\title{
Fabrication of fully suspended pyrolytic carbon string resonators for characterization of drug nano- and microparticles
}

Nguyen, Long Quang; Larsen, Peter Emil; Bishnoi, Shahana; Boisen, Anja; Keller, Stephan Sylvest

\section{Published in:}

Sensors and Actuators A: Physical

Link to article, DOI:

10.1016/j.sna.2019.02.002

Publication date:

2019

Document Version

Peer reviewed version

Link back to DTU Orbit

Citation (APA):

Nguyen, L. Q., Larsen, P. E., Bishnoi, S., Boisen, A., \& Keller, S. S. (2019). Fabrication of fully suspended pyrolytic carbon string resonators for characterization of drug nano- and microparticles. Sensors and Actuators A: Physical, 288, 194-203. https://doi.org/10.1016/j.sna.2019.02.002

\section{General rights}

Copyright and moral rights for the publications made accessible in the public portal are retained by the authors and/or other copyright owners and it is a condition of accessing publications that users recognise and abide by the legal requirements associated with these rights.

- Users may download and print one copy of any publication from the public portal for the purpose of private study or research.

- You may not further distribute the material or use it for any profit-making activity or commercial gain

- You may freely distribute the URL identifying the publication in the public portal 


\title{
Fabrication of fully suspended pyrolytic carbon string resonators for characterization of drug nano- and microparticles
}

Long Quang Nguyen ${ }^{1,2}$, Peter Emil Larsen ${ }^{1,3}$, Shahana Bishnoi ${ }^{1,2}$, Anja Boisen ${ }^{1,3}$ and Stephan Sylvest Keller ${ }^{1,2, *}$

1 DNRF and Villum Fonden Center for Intelligent Drug Delivery and Sensing Using Microcontainers and Nanomechanics, IDUN, Technical University of Denmark, 2800 Kgs. Lyngby, Denmark

2 National Centre of Nano Fabrication and Characterization, DTU Nanolab, Technical University of Denmark, 2800 Kgs. Lyngby, Denmark

3 Department of Health Technology, DTU Health Tech, Technical University of Denmark, 2800 Kgs. Lyngby, Denmark

* Corresponding author. Tel: +45 45255846. Email: suke@ dtu.dk

\begin{abstract}
Pyrolytic carbon is a promising material for fabrication of micro- and nanomechanical sensors due to the possibility to control both structural parameters and properties such as resonance frequency, residual stress and electrical conductivity. Here, we present a novel fabrication process for pyrolytic carbon string resonators suspended over a through-wafer-orifice. The final pyrolytic carbon string resonators had a length of 400 $\mu \mathrm{m}$, a width of $30 \mu \mathrm{m}$ and a thickness of $700 \mathrm{~nm}$. The string resonators were applied for characterization of nano- and microparticulate drug formulations using two different modes of operation. First, mass sensing on nanograms of nebulized or spray coated paracetamol micro- and nanoparticles was demonstrated. Then, absorption spectra of the drug were obtained by nanomechanical infrared spectroscopy (NAMIR). Finally, an efficient method for in situ regeneration of the sensor was demonstrated.
\end{abstract}

Keywords: pyrolytic carbon, mass sensing, IR spectroscopy, paracetamol, MEMS resonators, doubly-clamped beam.

\section{Introduction}

Microelectromechanical systems (MEMS) have been widely applied for sensing of different types of physical parameters, such as changes in mass, force and temperature. More specifically, MEMS resonators, which are based on tracking changes in resonance frequency, provide high sensitivity, small device size and simple readout 
methods without any analog-digital conversion. Based on the working principle, there are many types of MEMS resonators such as bulk mode, torsional mode or flexural mode resonators [1-3]. Bulk mode operation can be described as standing longitudinal waves while in the torsional mode, the dominant stress is shear-stress and the displacement produced is rotational. Flexural mode is representative of transverse standing waves, therefore the displacement in flexural modes is orthogonal to the bending stress in the structure. In this work, the flexural mode principle was selected. Based on the geometry, flexural mode resonators can be divided in three main categories of commonly used devices: i) cantilevers which are singly-clamped beams, ii) bridges which are doubly-clamped beams and iii) membranes. The cantilever-based resonators provide good sensitivity in force and mass sensing $[4,5]$. However, intrinsic stress gradients obtained in the cantilever material during fabrication often lead to significant out-of-plane bending. Furthermore, in terms of temperature and heat detection, cantilevers have some drawbacks due to the requirement of bi-material designs in order to track the change of temperature. Compared to that bridges and membranes can work as single material resonators measuring detuning of the resonance frequency. Bridges and membranes are quite compatible with many modes of detection such as mass, temperature and heat. However, bridges typically provide higher sensitivity in mass and heat sensing than membranes [6, 7]. The sensitivity of doubly-clamped beams can be further improved when the resonating structures are under high tensile stress and become string resonators [8]. As of today, most MEMS string resonators have been fabricated with Silicon Nitride ( $\mathrm{SiN}$ ) as the beam material. These SiN string resonators have been used for many applications such as temperature sensors [6, 7], mass sensors $[9,10]$ or even a combination of mass sensing with other techniques such as infrared (IR) spectroscopy to obtain an infrared sensor [11]. These nanomechanical IR (NAM-IR) sensors are a powerful tool to characterize many types of particles such as explosives molecules [12] and biological analytes [13]. Basically, when IR light is irradiated onto the resonators, the resonance frequency of the resonator changes depending on the IR absorption characteristics of the adsorbed molecules. From the recorded photothermal spectra, the adsorbed molecules can be identified.

Recently, carbon has become increasingly attractive as material for resonator sensors such as graphene pressure sensors [14] or carbon nanotube (CNT) mass sensors [15]. Among all those popular forms of carbon, pyrolytic carbon recently has been identified as a potential candidate for fabrication of MEMS sensors [16-19]. Basically, pyrolytic carbon can be obtained in the so-called C-MEMS process, where photoresist is pyrolyzed at high temperatures in an inert atmosphere [20,21]. Pyrolytic carbon is a promising material for fabrication of MEMS sensors due to the possibility to control both structural parameters and properties such as resonance frequency, residual stress 
and electrical conductivity. Recently, pyrolytic carbon has been used to fabricate string resonators with a simple fabrication process by combining lithography, dry etching techniques and pyrolysis [22]. This process has been further optimized for improved performance of pyrolytic carbon string resonators [23].

However, two main challenges have to be addressed to allow for the successful application of pyrolytic carbon string resonators for mass sensing and NAM-IR on nano- and microparticles. Both of them are related to the presence of an underlying Si substrate in previous designs of pyrolytic carbon string resonators released with a dry etch from the front side of the carrier substrate [24]: i) the Si substrate causes difficulties in depositing nano- and microparticles on the string resonators because the flow of liquid or gaseous samples is obstructed. Furthermore, the particles are deposited in the small gap between the string and the substrate which affects the free vibration of the string; ii) although the Si substrate is almost transparent for IR light, alignment of the sensor and the laser beam for NAM-IR measurement from the backside is very challenging. Therefore, a sensor based on pyrolytic carbon string resonators fully suspended over a through-wafer orifice has to be developed to allow for both optimal sampling of nano- and microparticles and NAM-IR measurements.

Here, we present a novel process for the fabrication of pyrolytic carbon string resonators based on the release of the structures from the backside of the substrate. The resulting fully suspended resonators were then evaluated as a tool for detecting drug nano- and microparticles. Paracetamol (Acetaminophen or N-acetyl-p-aminophenol) was chosen as a model analyte and two different deposition methods, nebulization and ultrasonic spray coating, where considered. Finally, the carbon string resonators were used as mass sensor for nanograms of drug nanoparticles and as chemical analysis tool for paracetamol microparticles with the NAM-IR technique.

\section{Materials and methods}

1. Fabrication process of pyrolytic carbon string resonators released from the backside

Figure 1 shows the fabrication process of pyrolytic carbon string resonators suspended over a through-wafer orifice. In this work, n-type double side polished Si wafers with a thickness of $350 \pm 15 \mu \mathrm{m}$ were used (Fig. 1a). This thickness was chosen to reduce the time for etching through the wafers. First, $200 \mathrm{~nm}$ of low stress Silicon Nitride (SiN) were deposited on both sides of the wafer in a Low Pressure Chemical Vapour Deposition (LPCVD) furnace (Fig. 1b). The SiN deposited on the top side of the wafer served as an etch stop layer during the later etching through the wafer due to its low etch rate in $\mathrm{KOH}$ solution. After deposition of the SiN layer $4 \mu \mathrm{m}$ of AZ5214E photoresist were patterned using standard UV photolithography with an exposure dose 
of $200 \mathrm{~mJ} / \mathrm{cm}^{2}$. The SiN layer on the backside was then etched by dry etch in an Advanced Oxide Etcher (AOE) from STS (Fig. 1d). The parameters for SiN etching

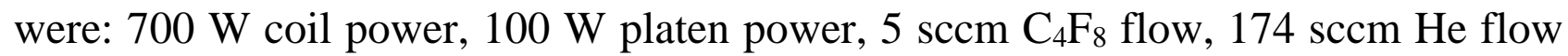
and $4 \mathrm{sccm} \mathrm{H}_{2}$ flow. The etching process was performed at 4 mTorr pressure at $0^{\circ} \mathrm{C}$ for 5 minutes to completely etch through the exposed SiN layer on the backside. Next, $\mathrm{Si}$ etching through the wafer was performed by using a combination of dry and wet etching (Fig. 1e). First, the Bosch process for dry etch of Si was applied by using an Advanced Silicon Etcher (ASE) from STS. In the etching step, a helium flow had to be used to keep the substrate temperature constant. As a consequence, the SiN layer on the front side of the wafer, which was in direct contact with the wafer chuck, easily broke when Si etching was performed through the complete thickness of the wafer. To avoid this, dry etching was run until $10 \mu \mathrm{m}$ of Si were left at the bottom of the etched openings. The dry etch of Si was performed for 53 minutes at $20^{\circ} \mathrm{C}$. The cycle time for etch and passivation step was 8 seconds and 5 seconds, respectively. For the etch step $230 \mathrm{sccm}$ of $\mathrm{SF}_{6}$ mixed with $23 \mathrm{sccm}$ of $\mathrm{O}_{2}$ were used while the passivation step only employed $120 \mathrm{sccm}$ of $\mathrm{C}_{4} \mathrm{~F}_{8}$. The power during the etch step was the combination of $2800 \mathrm{~W}$ coil power and $19 \mathrm{~W}$ of platen power. $1000 \mathrm{~W}$ coil power was used in the passivation step. The wet etching of the remaining Si was executed in $\mathrm{KOH}$ solution (28\%) at $80^{\circ} \mathrm{C}$ in 1 hour. After etching through the wafer only $200 \mathrm{~nm}$ thin SiN membranes were left to continue the process on the frontside. For the device layer, 6 $\mu \mathrm{m}$ thick SU-8 2005 were spin coated followed by solvent evaporation for $2 \mathrm{~h}$ and UV exposure with a dose of $200 \mathrm{~mJ} / \mathrm{cm}^{2}$ to pattern the string structures [23]. After a postexposure bake for at $50^{\circ} \mathrm{C}$ for $1 \mathrm{~h}$ and development in PGMEA (Fig.1f), an additional hard-bake for 1 hour at $120^{\circ} \mathrm{C}$ was introduced. After the SU-8 string resonator structures were defined, the SiN membrane was etched by a Reactive Ion Etcher (RIE) from STS (Fig. 1g). To completely remove the SiN below the SU-8, the etching process was performed from the backside of the wafer through the open window. The parameters of this etching step were: $8 \mathrm{sccm}$ of $\mathrm{CHF}_{3}, 43 \mathrm{sccm}$ of $\mathrm{N}_{2}, 50 \mathrm{~W}$ power, 38 mTorr pressure and an etching time of 10 minutes. The final step of the fabrication process was pyrolysis to transform the released SU-8 microstructures into pyrolytic carbon strings (Fig. 1h). The pyrolysis processes were conducted in a PEO-604 furnace manufactured by ATV Technology with the optimized parameters presented in our previous work [23]. First, the temperature was ramped to $200^{\circ} \mathrm{C}$ where it was held for 30 minutes. Then, the ramping was continued with a rate of $30^{\circ} \mathrm{C} / \mathrm{min}$ to the maximum temperature of $700^{\circ} \mathrm{C}$ which was held for 10 minutes. 


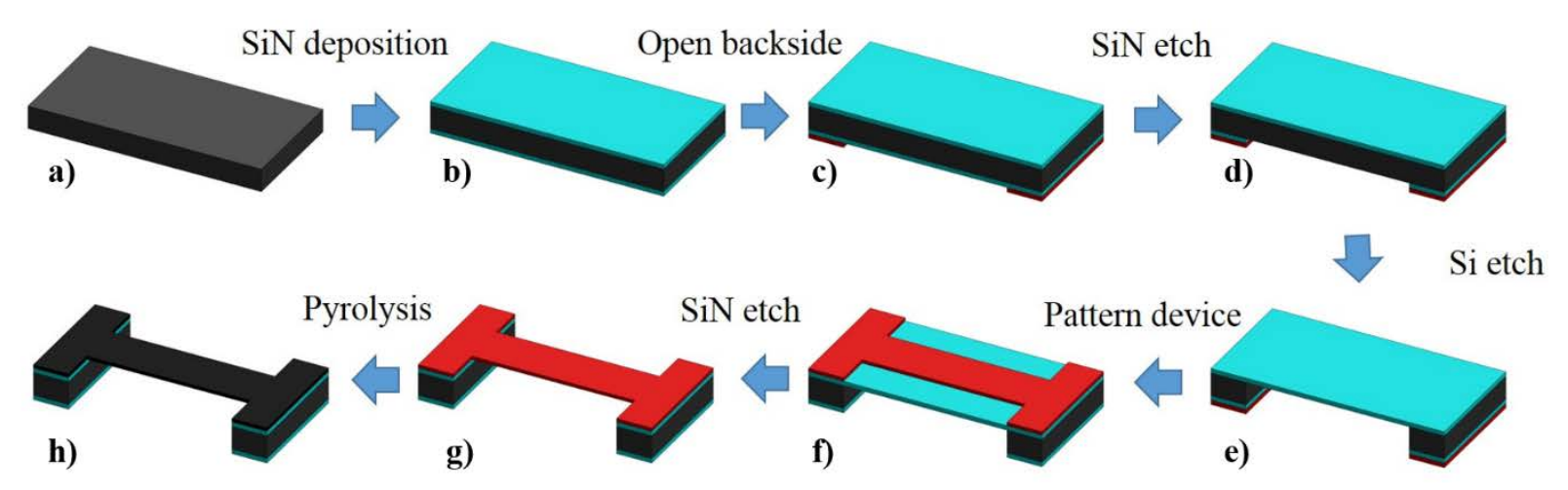

Figure 1: Fabrication process of pyrolytic carbon string resonators with backside opening window, a) Silicon wafer, b) SiN deposition, c) Photolithography to open window on backside, d) SiN etched from backside, e) Si etch through wafer, f) String patterning by SU-8 lithography, g) Etching of SiN membranes, h) Pyrolysis to obtain carbon string.

\section{Measurement of density and thickness of pyrolytic carbon}

For the calculation of the added mass, the density and thickness of the pyrolytic carbon string resonator material had to be determined. For this purpose, unpatterned SU-8 photoresist films were processed on 4 inch Si wafers with identical parameters as for the fabrication of the string resonators. The samples were weighed on a microbalance before spin-coating and after pyrolysis of the polymer films to obtain the mass of the pyrolytic carbon. The carbon layer was mechanically removed at several points across the wafer and the film thickness was determined using a stylus profilometer. The thickness of the actual resonators was measured using the same method.

\section{Preparation of paracetamol solutions}

Acetaminophen or paracetamol with laboratory analytical grade was purchased from Sigma Aldrich with molecular weight of $151.16 \mathrm{~g} / \mathrm{mol}$. Solutions with different concentrations of paracetamol were prepared by adding $1 \mathrm{mg}$ of paracetamol to different volumes of solvent. For the deposition of paracetamol nanoparticles methanol was used due to the high solubility of paracetamol in this solvent [25]. For deposition of microparticles the spray coating method required a high evaporation rate of the solvent and therefore ethanol was selected.

\section{Deposition of paracetamol nanoparticles}

The use of fibrous filters is a common method to clean air from micro- and nanosized particles. Here, this method was applied for deposition of paracetamol nanoparticles on the pyrolytic carbon string resonators functioning as a single filter-fiber [10]. The 
paracetamol particles were blown through the orifice in the wafer and captured by impaction on the suspended pyrolytic carbon string resonators. A dedicated system for this sampling method was designed (Figure 2). The filtered air supply (3074B, TSI, Shoreview, MN, USA) removed oil, liquid droplets and fine particles from the incoming air. The pressure was controlled by a built-in gas regulator valve. Paracetamol nanoparticles were generated using a jet nebulizer (3076, TSI, Shoreview, MN, USA).

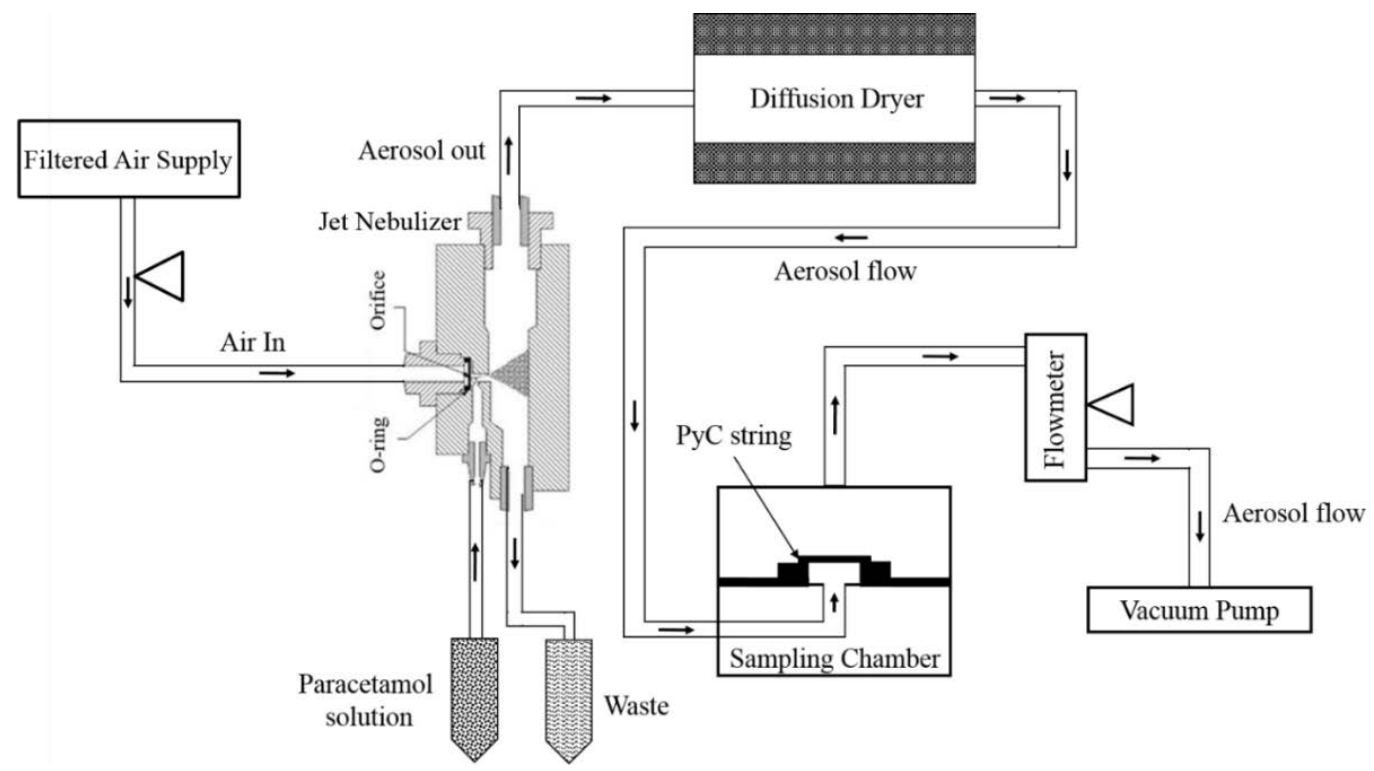

Figure 2: Schematic describing the jet nebulizer system for deposition of paracetamol nanoparticles on pyrolytic carbon (PyC) strings.

In this system, air was pressed through an orifice which had a diameter of $0.34 \mathrm{~mm}$ to generate a high velocity jet. Paracetamol solutions with different concentrations were drawn into the nebulizing section through a vertical passage and nebulized by the jet. Large droplets were removed by impaction on the wall opposite to the jet and the wasted liquid was drained at the bottom of the nebulizer assembly block. Aerosol was extracted from the device through the fitting at the top and travelled through the diffusion dryer filled with silica beads. Then the aerosol was directly introduced into the aluminum chamber where the pyrolytic carbon string resonator was suspended over the orifice placed perpendicularly to the nanoparticle flow. At the outlet of the chamber, suction was applied by vacuum. This increased the nanoparticle velocity and allowed to regulate the sampling efficiency on the pyrolytic carbon string resonators. The air flow was controlled by a valve (VFA-22-SSV from Dwyer, Michigan, IN, USA) and measured by a flowmeter (4100 from TSI, Shoreview, MN, USA).

5. Deposition of paracetamol microparticles 
For the deposition of paracetamol microparticles, an Exacta Coat Ultrasonic Spray System (Sonotek, USA) was used in a setup similar to the one described earlier [26]. The tip of the ultrasonic atomizer nozzle (Impact, Sonotek, USA) was actuated at a frequency of $120 \mathrm{kHz}$ with a run power of $1.3 \mathrm{~W}$. The parameters of the spray process were optimized to obtain a high efficiency of harvesting of the drug microparticles on the pyrolytic carbon string resonators. For this purpose, paracetamol solution with $2 \%$ in ethanol was placed in a syringe and pumped through the nozzle at a rate of 0.1 $\mathrm{mL} / \mathrm{min}$ using a syringe pump. Pressurized air was connected to the beam shaping unit of the nozzle and the air flow was controlled by a pressure controller set to 20 mbar. The gas flow and the distance between nozzle and substrate allowed control of the diameter and shape of the spray coating beam. In this work, the distance from the nozzle tip to the substrate was fixed at $4 \mathrm{~cm}$. During the experiment, the temperature of the substrate was kept constant at $25^{\circ} \mathrm{C}$ using the integrated hotplate. Using this method, the samples were spray coated for a number of spray coater passes varying from 20 to 200. The movement of the nozzle was controlled by an $x-y-z$ stage. The spray coating was performed in a continuous path at a velocity of $20 \mathrm{~mm} / \mathrm{s}$. The nozzle was moving across a selected area in a snake like pattern with a distance of $4 \mathrm{~mm}$ between the lines. To ensure uniform coverage across the sample, every second pass of spray coating was performed with a $2 \mathrm{~mm}$ offset.

\section{Characterization setup}

Before and after deposition of drug micro- or nanoparticles, the pyrolytic carbon string resonators were placed in a vacuum chamber which was pumped down to below $10^{-5}$ mbar. The chip was actuated by a ring piezo crystal placed below it. The resonance frequency and quality factor (Q) of the pyrolytic carbon string resonators were optically measured with a MSA-500 laser Doppler vibrometer from Polytec and a lock-in amplifier from Zurich Instruments.

First, the mass sensing method was used to characterize the deposition of paracetamol nano- and microparticles on the string resonators. The fundamental resonance frequency of the resonators was determined by means of phase-locked loop with a lockin amplifier before and after drug deposition. Based on the shift of the resonance frequency, the mass of the deposited paracetamol was determined.

In order to chemically analyze the sample, NAM-IR measurements were performed. For this purpose, IR radiation emitted by a tunable Quantum Cascade Laser (QCL) was focused on the backside of the carbon string with a parabolic mirror [27]. The laser duty cycle was set to $0.3 \%$ (pulse duration $50 \mathrm{~ns}$ ), and the repetition rate was set to 100 
$\mathrm{kHz}$. The IR wavenumber was swept with a $100 \mathrm{~ms}$ dwell time and a $5 \mathrm{~cm}^{-1}$ step size from $768 \mathrm{~cm}^{-1}$ to $1953 \mathrm{~cm}^{-1}$.

At specific wavelengths, the resonance frequency of the carbon string was detuned due to the heat absorbed by the drug molecules. Based on the tracking of the resonance frequency, the photothermal spectra was recorded with both a blank carbon string as a reference and a string with a deposited sample. By subtracting those two spectra from each other, the absorption spectrum of the sample molecule was extracted. Figure 3 shows a schematic of the setup for characterization of pyrolytic carbon string resonators.

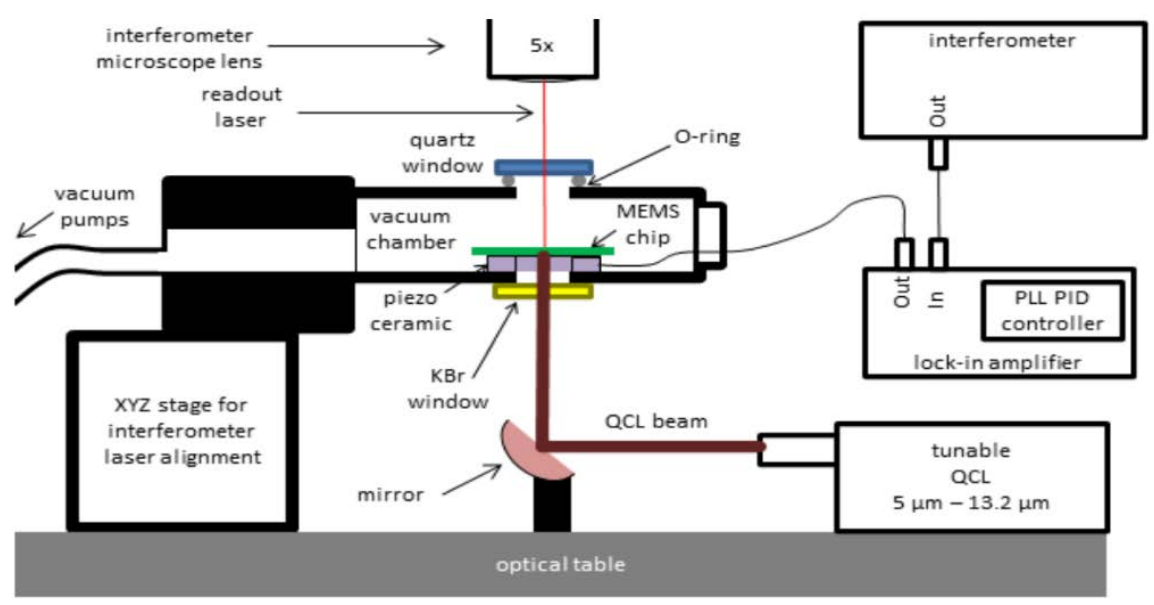

Figure 3: Schematic of setup for Nanomechanical Infrared (NAM-IR) Spectroscopy with interferometric readout and Quantum Cascade Laser (QCL) for excitation [27]

\section{Results and discussion}

1. Fabrication and characterization of the pyrolytic carbon string resonators

Doubly-clamped pyrolytic carbon resonators were successfully fabricated with the novel process based on a release by etching from the backside of the substrate. Figure 4 shows a $400 \mu \mathrm{m}$ long pyrolytic carbon string resonator fully suspended over a through wafer orifice fabricated at $700^{\circ} \mathrm{C}$ pyrolysis temperature. Because of mass loss during the pyrolysis process, the width of the string was approximately $20 \%$ lower $(25 \mu \mathrm{m})$ compared to the one of the SU-8 structures before pyrolysis $(30 \mu \mathrm{m})$ and the edge of the string was bending upwards because of residual stress gradients. However, this deformation did not seem to affect the performance of the sensors. The thickness of the actual resonators was approximately $700 \mathrm{~nm}$. The calculated density of pyrolytic carbon was $1.20 \pm 0.01 \mathrm{~g} / \mathrm{cm}^{3}$. 

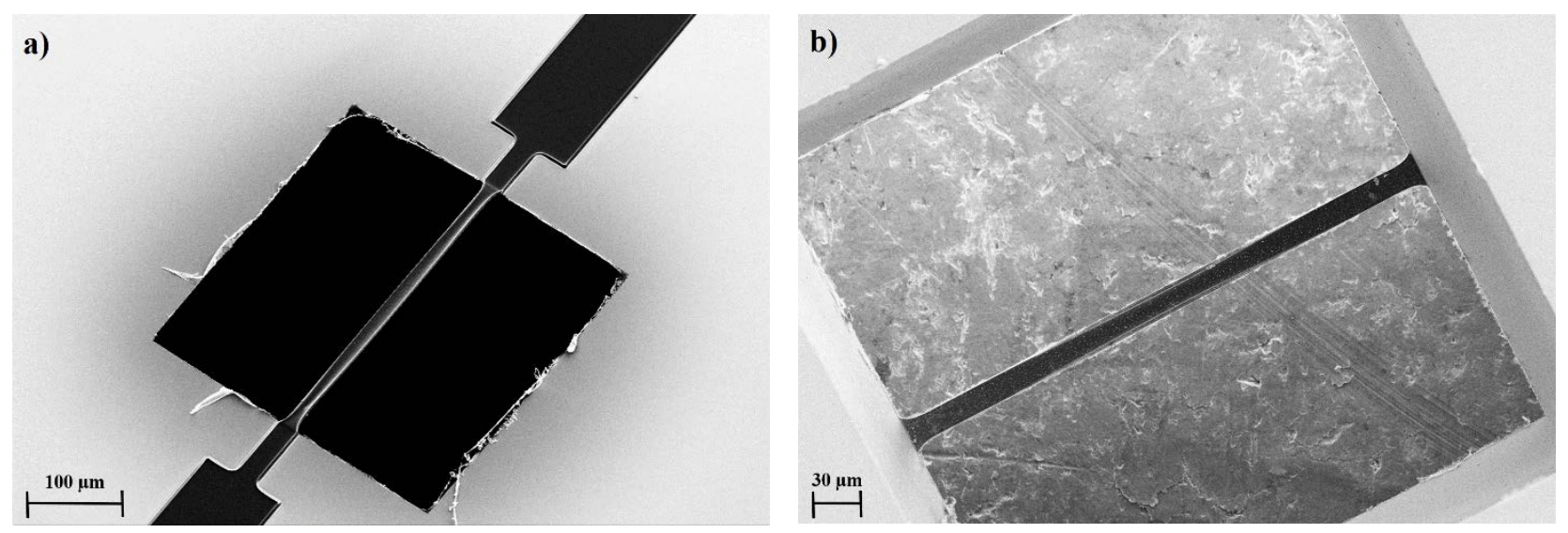

Figure 4: Pyrolytic carbon string resonator $(400 \times 30 \mu \mathrm{m})$ fully suspended over a through wafer orifice imaged from a) the front side and $b$ ) the backside of the wafer

After fabrication, the resonant behavior of the fully suspended doubly-clamped pyrolytic carbon resonators was characterized with the laser Doppler vibrometer system. Figure 5 shows the resonance frequencies and $\mathrm{Q}$ factors of beams with different lengths ranging from $300 \mu \mathrm{m}$ to $700 \mu \mathrm{m}$ while the nominal width of the structures varied from $15 \mu \mathrm{m}$ to $30 \mu \mathrm{m}$. For string resonators, the resonance frequency $\mathrm{f}_{\mathrm{n}}$ of the nth mode is given as [4]:

$$
f_{n}=\frac{n}{2 L} \sqrt{\frac{\sigma}{\rho}}
$$

Where $\sigma$ is the tensile stress, $L$ is the length of the string and $\rho$ is the density of the string resonator material. The observed linear dependency of resonance frequency on 1/L (equation 1) confirmed that the devices had a string-like behavior. Compared with carbon string resonators released from the front side [23], the resonance frequencies for devices with identical nominal dimensions released from the backside were slightly higher. This can be explained by the different etching processes used during fabrication where the isotropic etch in [23] resulted in longer strings compared to the anisotropic etch in the present work. As in earlier experiments [23], the highest Q factors were achieved with strings having a length of $400 \mu \mathrm{m}$. This can be explained by the dominance of clamping losses (Qclamping) for shorter string resonator $(<400 \mu \mathrm{m})$ that led to a decrease of the $\mathrm{Q}$ factor. For longer strings $(>400 \mu \mathrm{m})$ the reduction of the $\mathrm{Q}$ factor is probably related to an increase of intrinsic damping ( $\left.Q_{\text {intrinsic }}\right)$ which could be caused by internal friction between graphitic and amorphous regions in the pyrolytic carbon. For that reason, the $400 \mu \mathrm{m}$ long and $30 \mu \mathrm{m}$ wide pyrolytic carbon string resonators were used for all further experiments. 

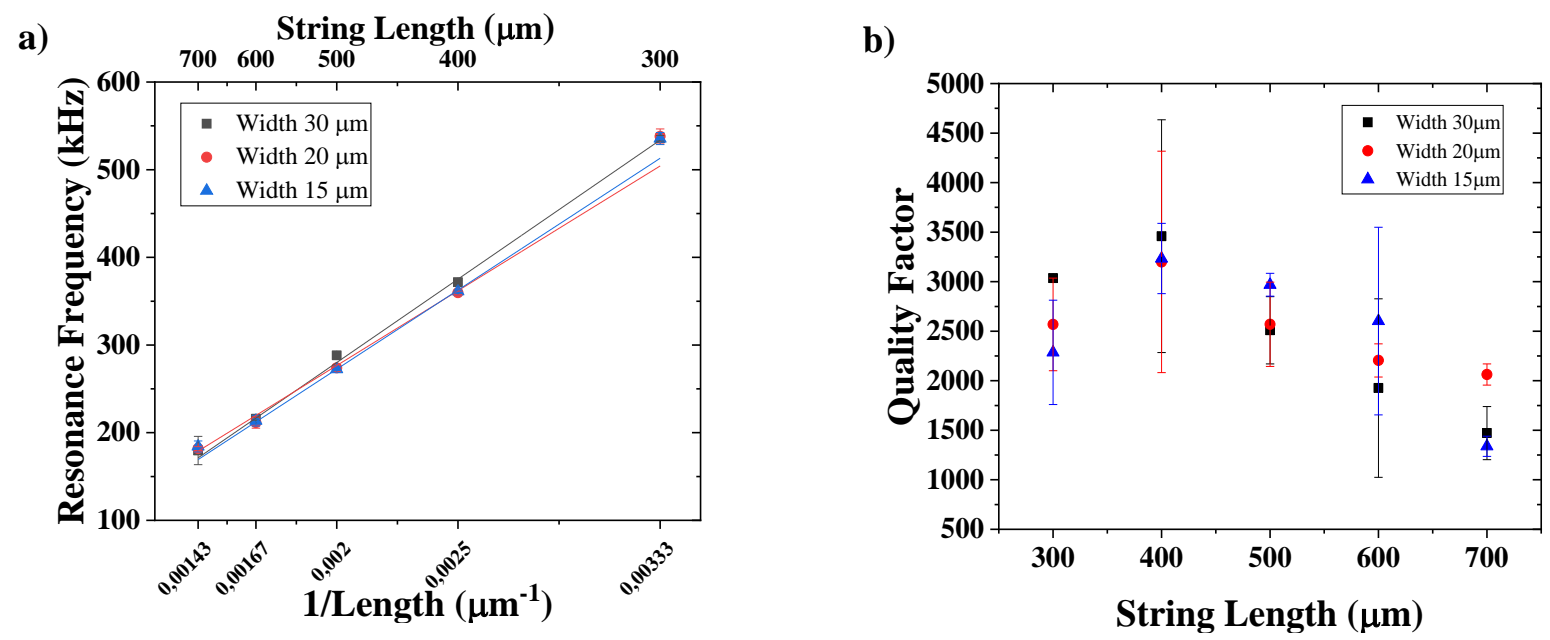

Figure 5: Resonance frequency (a) and Q factor (b) of fully suspended pyrolytic carbon string resonators. Data is presented as mean $\pm S D(n=5)$.

2. Deposition of paracetamol nanoparticles on pyrolytic carbon resonators

First, it was evaluated if the through wafer orifice allowed controlled deposition of nanoparticles on the fabricated fully suspended pyrolytic carbon string resonators. For this purpose, a jet nebulizer was used to convert paracetamol solutions in methanol into nanoparticle aerosols. This aerosol was directed through the orifice in the chip with the carbon string acting as a filter fiber slowly accumulating particles on its backside due to diffusion or inertial impaction [10]. In the diffusion regime, Brownian motion increases the probability of nanoparticle adsorption on the string when those are passing by on an intercepting streamline. In the inertial regime, the nanoparticles are unable to adjust quickly enough to abruptly changing streamlines and impinge on the string. For a volumetric flow of $0.2 \mathrm{~L} / \mathrm{min}$ used in this work, the flow velocity in the through-wafer orifice with a side length of $400 \mu \mathrm{m}$ was estimated to approximately 21 $\mathrm{m} / \mathrm{s}$. According to Schmid et al. it was therefore expected that inertial impaction was the dominant mechanism for nanoparticle deposition on the carbon string resonators [10]. Figure 6 shows an SEM image of the bottom side of the fully suspended pyrolytic carbon string resonator after nanoparticle sampling for $10 \mathrm{~min}$. The white dots indicate the paracetamol nanoparticles deposited on the carbon string. The relatively uniform distribution of nanoparticles on the surface confirmed that deposition was mainly due to impaction. 


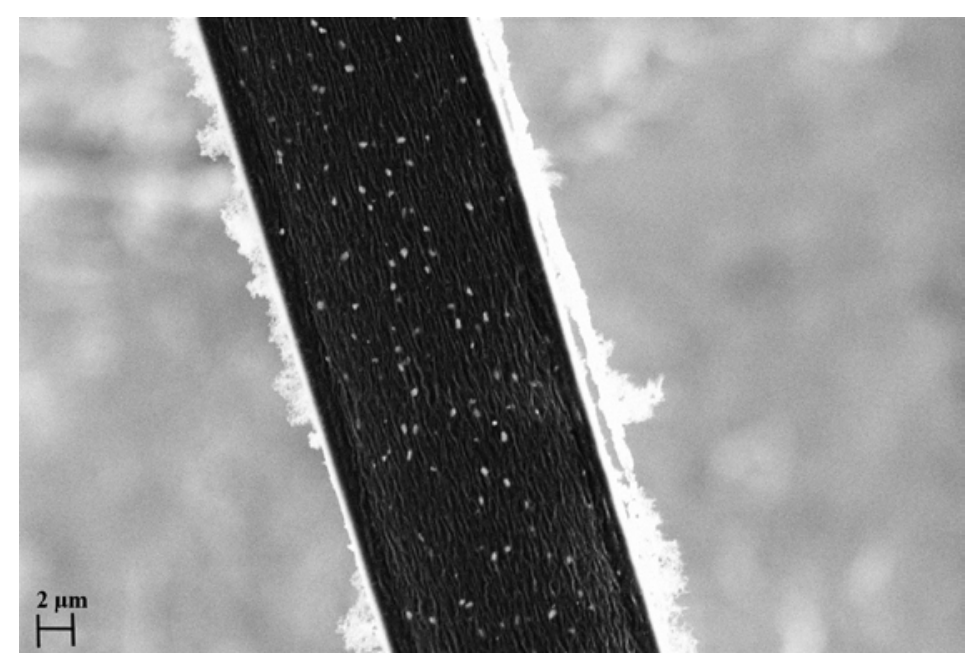

Figure 6: SEM image of bottom side of fully suspended pyrolytic carbon string resonator after nanoparticle sampling.

3. Mass sensing with paracetamol nanoparticles

Measurements of resonance frequency before and after nanoparticle deposition were used to determine the deposited mass and estimate the limit of detection for mass sensing with the pyrolytic carbon resonators. In mass sensing, a string resonator is generally referred to as beam-based mass sensor. For this type of sensors, it is possible to represent the beam-dynamics of an individual resonance mode with that of a simple harmonic oscillator:

$$
f_{0}=\sqrt{\frac{k_{e f f}}{m_{e f f}}}
$$

Where $k_{\text {eff }}$ and $m_{\text {eff }}$ are the effective spring constant and effective mass of the string resonator. Equation (2) shows that the resonance frequency depends on the mass.

The deposited mass $\Delta \mathrm{m}$ can be calculated based on the shift of resonance frequency assuming that $k_{\text {eff }}$ is constant before and after deposition [8]:

$$
\Delta m=m_{0}\left(\left(\frac{f_{0}}{f_{0}-\Delta f}\right)^{2}-1\right)
$$

This assumption is reasonable based on the fact that according to equation (1), for devices with a string-like behavior before and after deposition, the resonance frequency is independent of the spring constant.

The resonance frequency of the resonators before and after sampling were extracted using the Laser Doppler vibrometer setup. The resonance frequency decreased after nanoparticle deposition due to the increase in mass. The mass of the deposited sample was calculated using the measured relative frequency shift and equation (3). Figure $7 \mathrm{a}$ 
shows the influence of the sampling time on relative frequency shift for paracetamol solutions at concentrations of $2 \mathrm{mg} / \mathrm{mL}$. The results indicated a linear correlation for the first 10 minutes of sampling. After this, the increase of resonance frequency shift was less prominent which could be explained by possible competition between deposition and removal of nanoparticles from the string surface due to impaction.

Next, the dependency of relative frequency shift on drug concentration in the nebulized solution was investigated. For this purpose, solutions of paracetamol with different concentrations were prepared, followed by 10 minutes sampling with the jet nebulizer. Figure $7 \mathrm{~b}$ shows that as the concentration was increased more nanoparticles were collected on the carbon string. Due to the change in mass, the relative frequency shift increased linearly with the increase of concentration.

In mass sensing applications of string resonators, the limit of detection is defined by the noise in the measurement. To estimate the noise, Allan Deviation measurements were performed with the fully suspended pyrolytic carbon string resonator (Supporting information). The minimum detectable fractional frequency shift $(\mathrm{SNR}=3)$ was determined as $120 \mathrm{ppm}$. Using equation (3), the limit of detection was calculated as $1.61 \mathrm{pg}$ and the mass sensitivity as $75 \mathrm{ppm} / \mathrm{pg}$.

a)

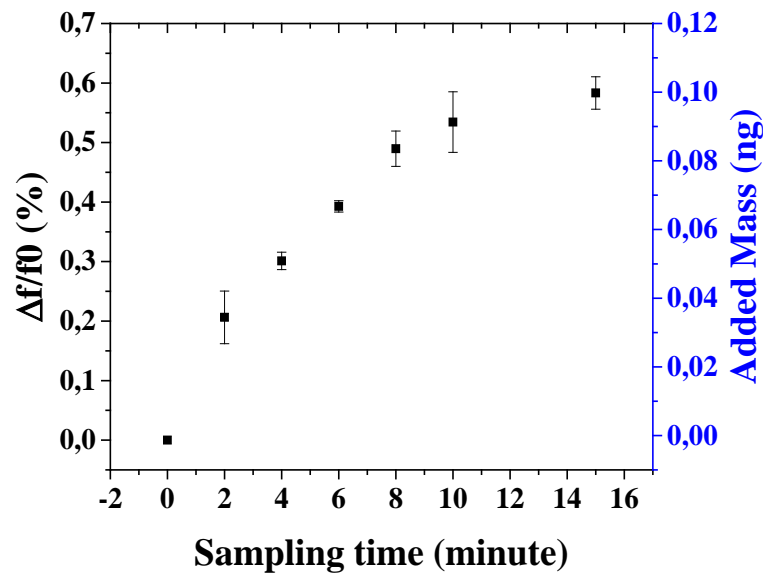

b)

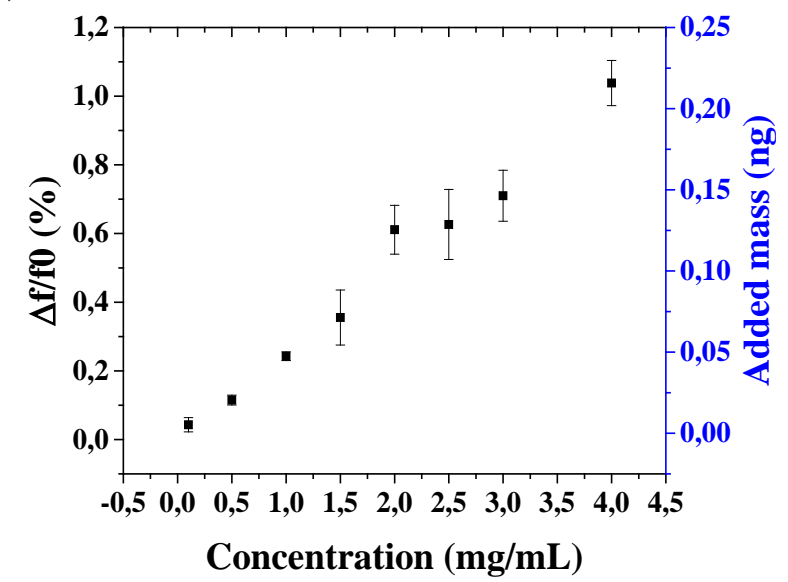

Figure 7: Dependency of relative frequency shift and calculated added mass on (a)

sampling time for paracetamol solution of $2 \mathrm{mg} / \mathrm{mL}$ and (b) concentration of paracetamol solution for 10 minutes sampling time. Data is presented as mean \pm SD $(n=5)$.

4. Spray coating of paracetamol microparticles on pyrolytic carbon resonators

The amount of drug deposited by jet nebulization ( $<1 \mathrm{ng}$ ) was unfortunately insufficient to evaluate NAM-IR as a second possible mode of operation for the pyrolytic carbon string resonators. Therefore, spray coating was considered as a second deposition method. Paracetamol solution with $0.5 \mathrm{wt} . / \%$ of paracetamol in ethanol was 
prepared and spray coated on the top surface of the pyrolytic carbon string resonators. Figure $8 \mathrm{a}$ and $8 \mathrm{~b}$ show the fully suspended pyrolytic carbon string resonators after 20 spray passes and 200 spray passes, respectively. Compared to the nebulizer, spray coating resulted in deposition of microparticles on the string resonators.
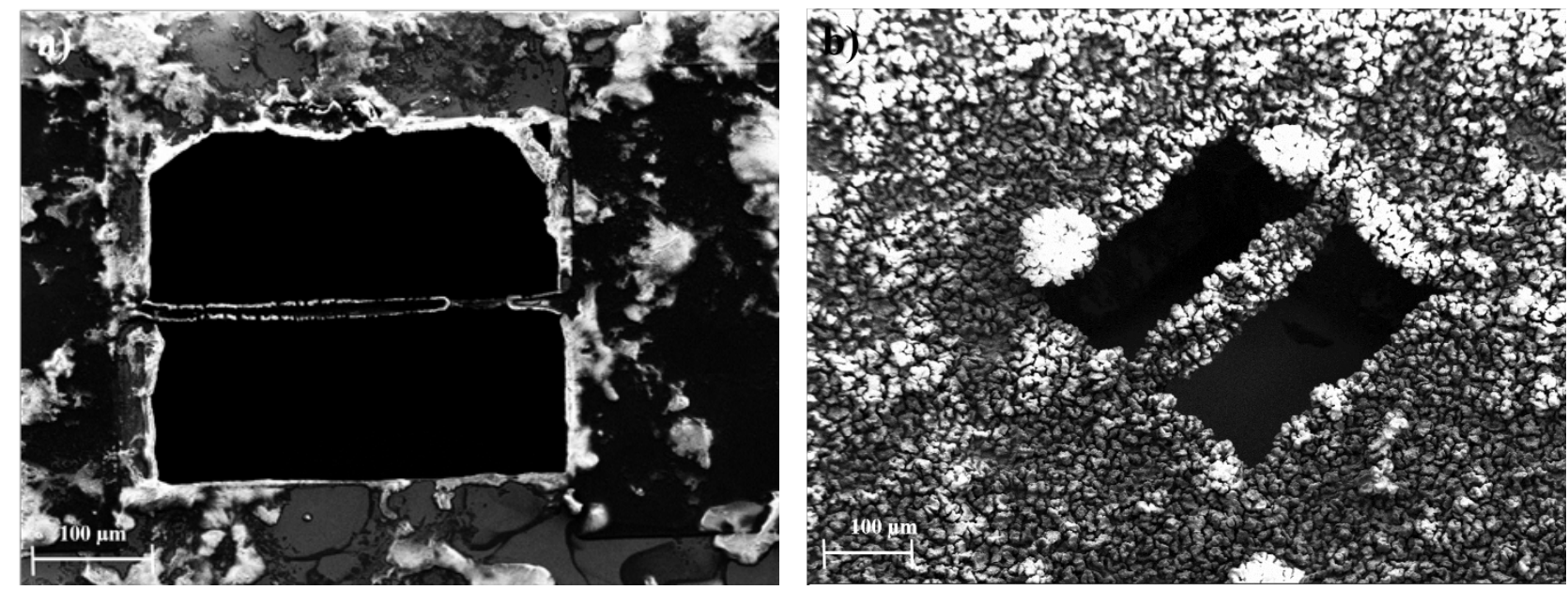

Figure 8: Fully suspended pyrolytic carbon string resonators after (a) 20 spray passes and (b) 200 spray passes.

The results in Figure 9a show a linear dependency of the frequency shift on the number of spray passes due to the increase of mass on the strings if the number of spray passes was below 100. For more than 100 spray passes, the change of resonance frequency shift was smaller. The non-linear increase of the frequency shift was probably explained by the fact that the mass of deposited drug microparticles was comparable or larger than the mass of the pyrolytic carbon string. Figure 9b shows the added mass calculated based on the resonance frequency shift. In order to verify the added mass deposited on the pyrolytic carbon strings by the spray coating method, the thickness of the spray coated paracetamol layers was measured with a stylus profilometer. Figure $9 \mathrm{~b}$ shows the mass added on the strings estimated based on the thickness measurements, the string dimensions and assuming a paracetamol density of 1.26 $\mathrm{g} / \mathrm{cm}^{3}$. The thickness of the paracetamol films and thereby the estimated added mass increased linearly with the number of spray passes. Figure $9 \mathrm{~b}$ demonstrates excellent correlation between the added mass calculated using the measured frequency shift and the added mass estimated using the thickness measurements. 
a)

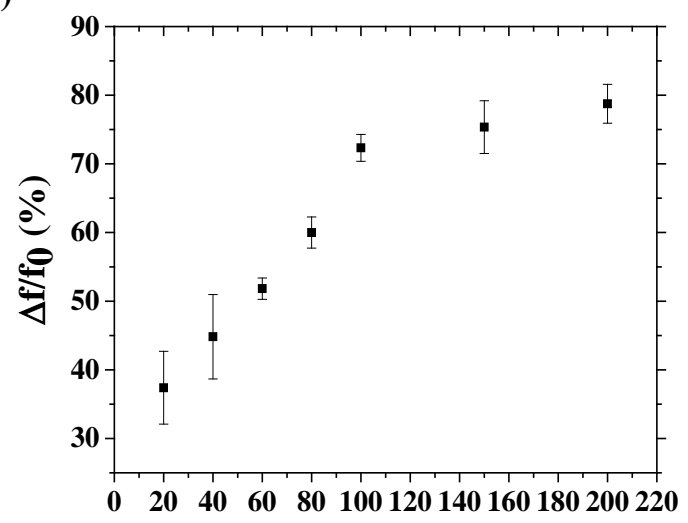

Number of spray pass b)

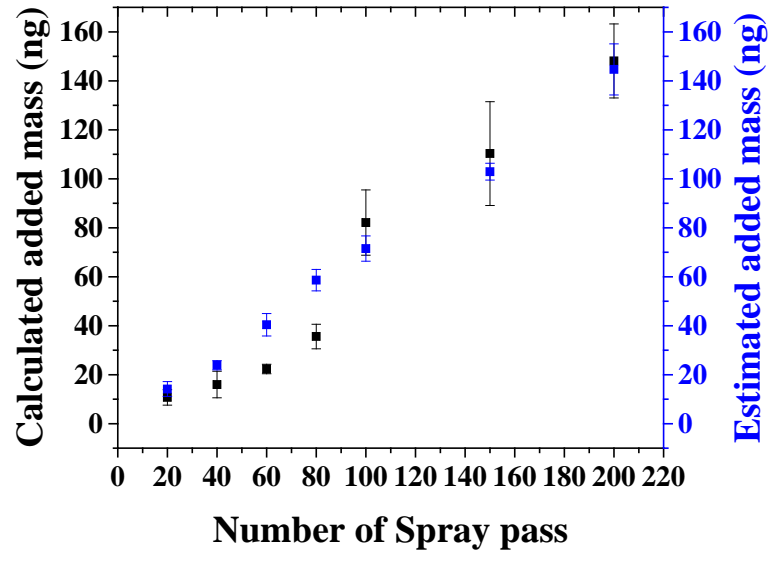

Figure 9: Dependency of relative frequency shift (a) and added mass (b) on number of spray passes.

\section{NAM-IR analysis of paracetamol microparticles}

The pyrolytic carbon string resonators fully suspended over the through wafer orifice were evaluated for identification of the drug using NAM-IR. In this method, the string resonators are used as thermal sensors monitoring temperature changes by tracking the detuning of the resonance frequency $[6,7,11]$. When the sensor is exposed to IR light of specific wavelengths, molecular vibrations in the molecules of either the sensor or the sample material can be excited. The dissipation of this absorbed energy generates heat, which is transferred to the string sensor. The resulting thermal expansion of the system leads to a small decrease in tensile stress which can be measured because of its effects on the resonance frequency as described by equation (1). If the string is heated in the center, the magnitude of the resonance frequency shift is maximized. The relative resonance frequency shift of the mechanical string resonator as the function of power $\mathrm{P}$ absorbed in the center of the string is given by [24]:

$$
\frac{\Delta f}{f_{0}}=-\frac{\alpha E}{\sigma k} \frac{L}{16 A} P
$$

Where $\mathrm{E}$ is the Young's modulus, $\alpha$ is the coefficient of thermal expansion (CTE), $\sigma_{0}$ is tensile pre-stress, and $\mathrm{k}$ is the thermal conductivity of the string material. $\mathrm{L}$ is the length and $\mathrm{A}$ is the cross sectional area of the string. This equation clearly shows that the relative frequency shift of the resonator is proportional to the absorbed power. Therefore, by tracking the resonance frequency shift while sweeping the wavelength of the excitation laser, the resulting recorded photothermal spectra yield an IR absorption spectra characteristic for the sample.

The string resonators with paracetamol microparticles were used for NAM-IR experiments. IR light from the QCL was focused on both blank references and string 
resonators with drug sample. The through wafer orifice provided easy access for the alignment of the laser from the backside. At specific wavelengths, the resonance frequency of the carbon string was detuned due to the heat absorbed by the drug molecules.

In order to obtain characteristic spectra from the target analyte, some signal processing was necessary. Figure 10 includes a step-by-step representation of this process. To obtain the raw data, the resonance frequency of the carbon string resonator was tracked using a phase-locked loop (PLL) while sweeping through the different IR wavelengths for both the blank reference and the string with drug sample, respectively (see Figure 10a). Next, the QCL sweep was cropped out and a baseline was calculated, taking heating of the entire chip throughout the experiment into account. Figure 10b shows baseline-corrected relative frequency shift for both the blank and the sampled string. The next step was subtraction of the signal from the blank string (absorption due to pyrolytic carbon and fabrication residues) from the experimental data to obtain the pure paracetamol IR absorption signal. However, since the alignment of the QCL is a manual process, the absolute IR laser power that reaches the sensor will be different for every experiment. To account for this, the blank signal was scaled to the experimental data before it was subtracted. The scaled blank signal is shown as the black curve in Figure 10b. It was scaled by the difference in relative frequency shift when the QCL was turned off (see Figure 10a). The difference of the two curves is shown in Figure 10c. Additionally, the corresponding QCL wavenumbers are interpolated. As a final signal processing step the QCL laser power spectrum had to be taken into account (see Figure 10d). By normalizing the subtracted signal according to the power spectrum the NAM-IR spectrum in Figure 10e was obtained. 
a)

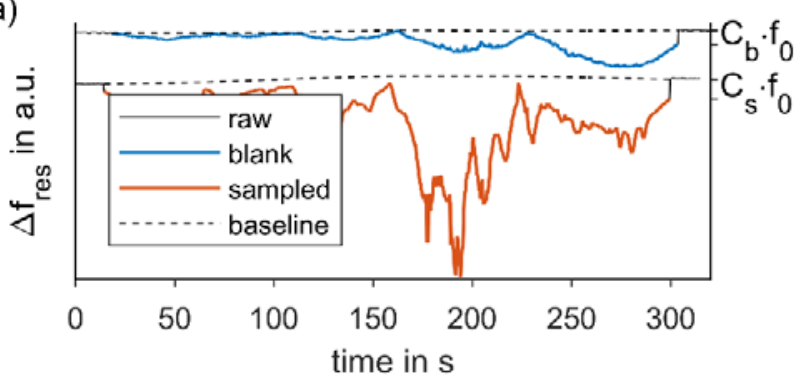

c)

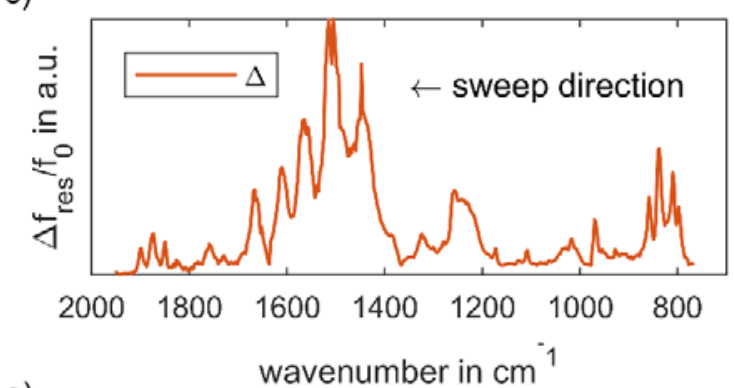

b)

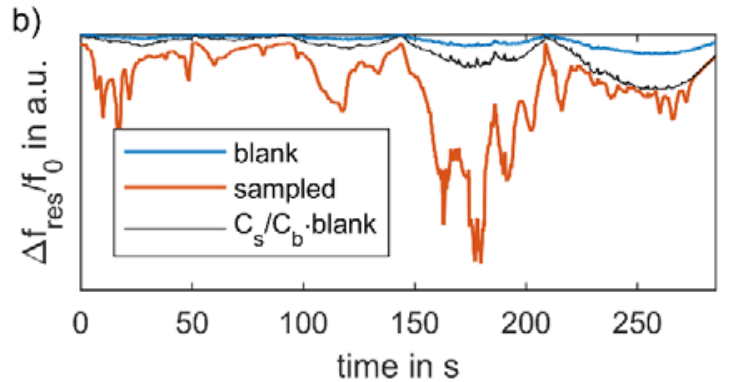

d)

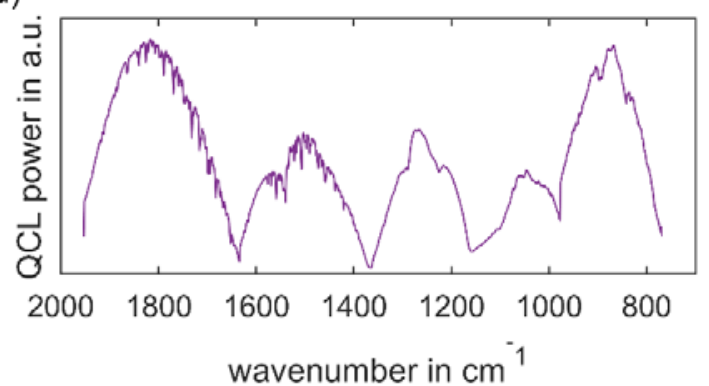

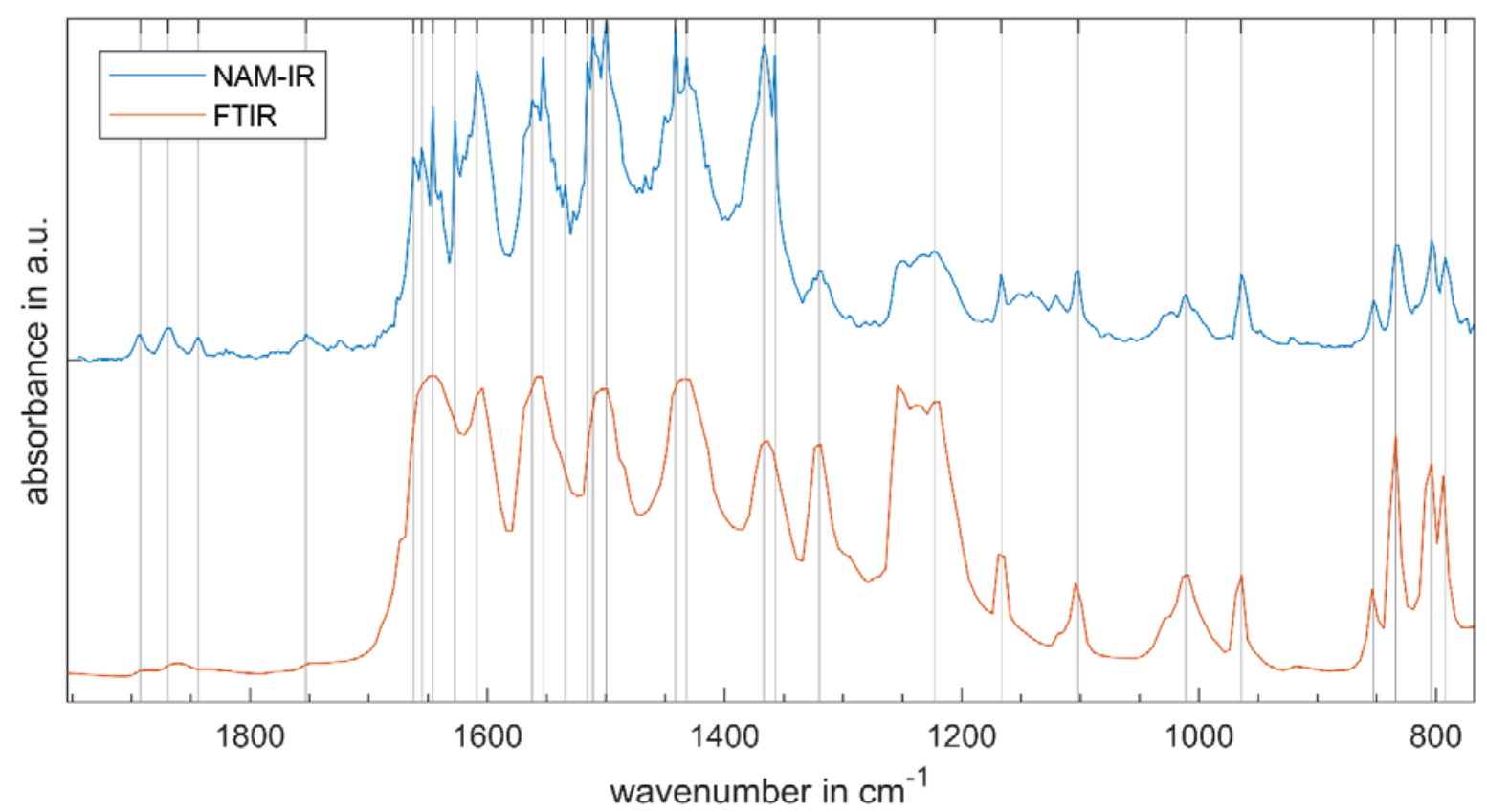

Figure 10: NAM-IR data analysis of blank and paracetamol coated resonator: a) raw data, b) scaled signal, c) subtraction of reference and sample signals, d) QCL spectrum and e) final NAM-IR results in direct comparison with FTIR spectra of paracetamol

The data shown here were obtained with a pyrolytic carbon string coated with 60 spray passes. Figure 10e compares the IR absorption spectrum of paracetamol from NAMIR with a reference Fourier-transform infrared spectroscopy (FTIR) spectrum obtained with several $\mu \mathrm{g}$ of drug sample [25]. The following characteristic peaks of paracetamol were detected with the NAM-IR method (798.2, 809.9, 840.1, 858.7, 970.4, 1016.9, 
1107.6, 1172.8, 1228.6, 1326.3, 1363.5, 1372.8, 1438, 1447.3, 1505.4, 1517.1, 1521.7, 1540.3, 1558.9, 1568.2, 1614.8, 1633.4, 1652, 1661.3, 1668.3, 1759, 1849.7, 1875.3, and $1898.6 \mathrm{~cm}^{-1}$ ). The results demonstrate that the NAM-IR method is able to identify the same characteristic peaks as FTIR. Furthermore, the peaks observed with NAM-IR are sharper than the peaks in the FTIR spectra. This is probably due to the low thermal mass of pyrolytic carbon as it earlier has been reported for a similar application of bismuth ferrite nanowire resonators [29].

\section{In situ regeneration of pyrolytic carbon string resonator}

The high absorption coefficient of the pyrolytic carbon for visible light in combination with the small dimensions of the string resonators potentially enable heating of the structures by the readout laser from the interferometer. The local heating achieved by the readout laser can be exploited to regenerate the paracetamol coated sensor. Figure 11 shows the pyrolytic carbon string before and after in situ regeneration with the readout laser. The temperature increase of the string obtained at the maximum laser power of $250 \mu \mathrm{W}$ was estimated to approximately $15^{\circ} \mathrm{C}$ (Supporting information). This means that the observed regeneration is not due to evaporation of the drug because this would require much higher temperatures. More probably, the removal of the spray coated paracetamol microparticles by the laser is a result of different thermal expansion coefficients between pyrolytic carbon and paracetamol which led to delamination of the drug particles from the carbon. The NAM-IR analysis of pyrolytic carbon string resonators after regeneration confirmed that most paracetamol was removed from the string resonator. Only a weak signal from the drug was still detected probably due to sample residues near the edges of the pyrolytic carbon strings.
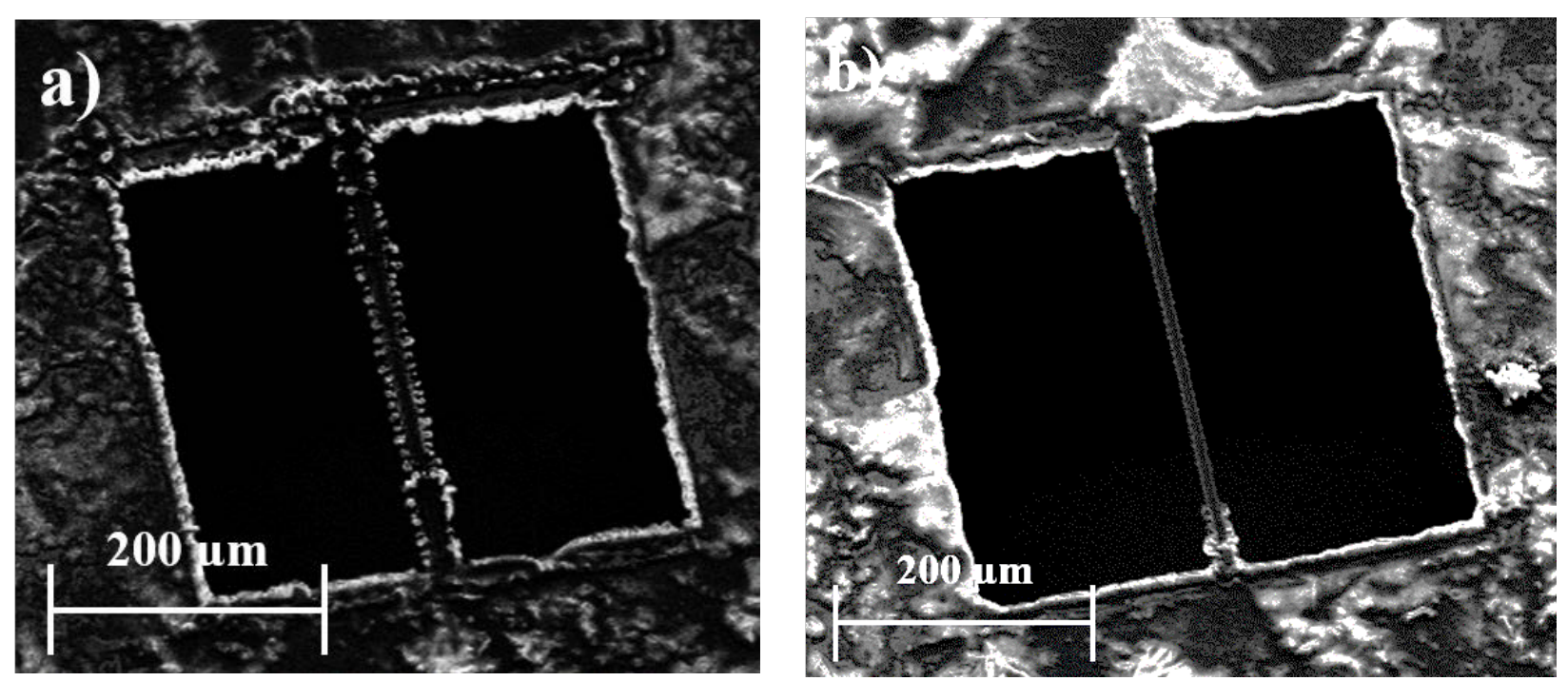

Figure 11: Pyrolytic carbon string coated with paracetamol microparticles before (a) and after (b) in situ regeneration by readout laser. 


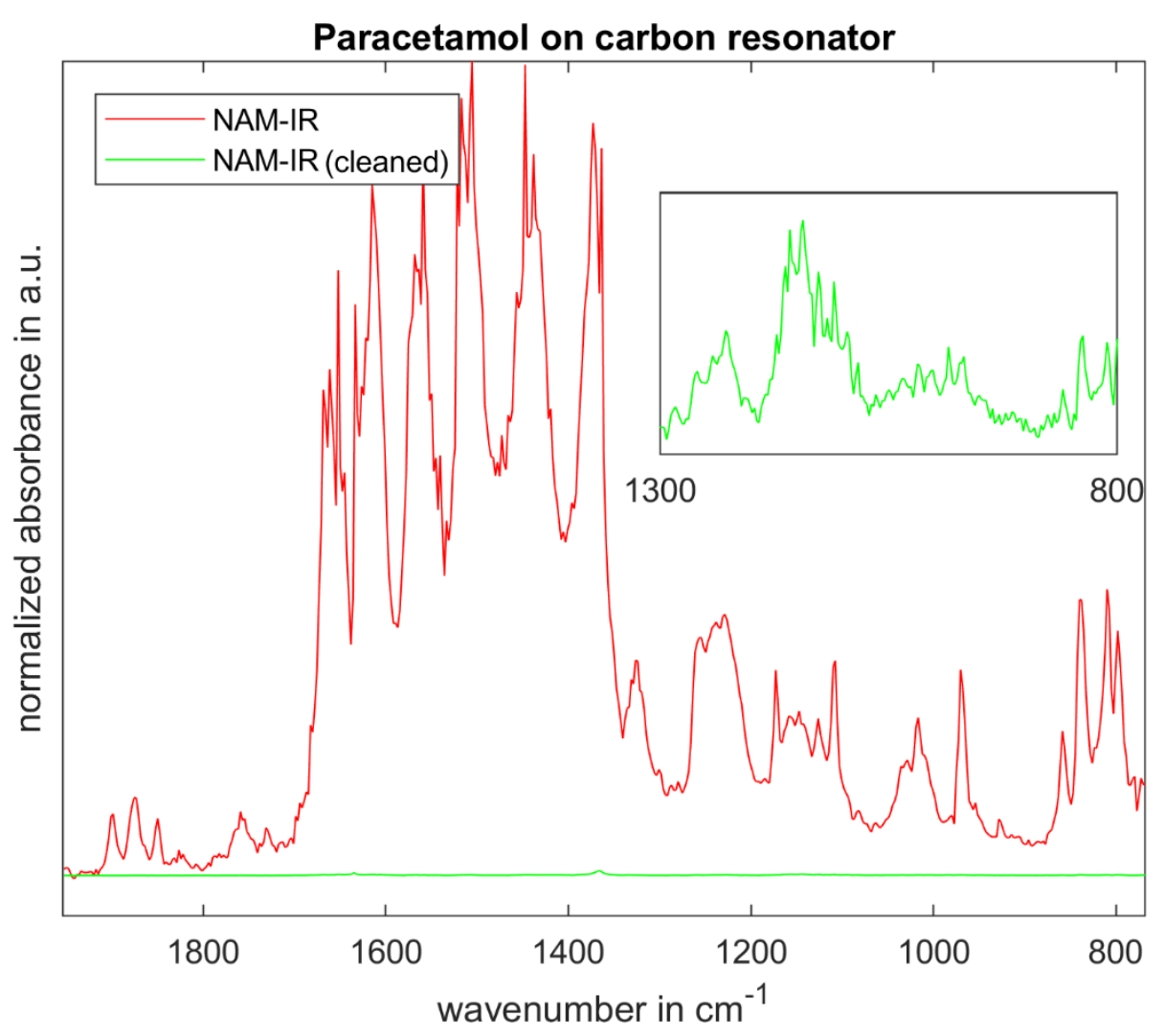

Figure 12: Normalized NAM-IR spectra of the pyrolytic carbon string resonator before (red) and after in situ regeneration (green).

\section{Conclusions}

In conclusion, pyrolytic carbon string resonators fully suspended over a through wafer orifice were successfully designed and fabricated. This novel configuration allowed deposition of paracetamol on the carbon string resonators using two methods: A jet nebulizer for nanoparticle deposition and spray coating for microparticle deposition. The deposited amount of paracetamol was determined by the resonant mass sensing principle to be in the range of 10-100 pg (jet nebulizer) to several ng (spray coating). We also performed NAM-IR spectroscopy with pyrolytic carbon string resonators for chemical identification of paracetamol drug particles. An advanced protocol for signal processing was implemented due to the IR absorption of the carbon resonator itself. We demonstrated that the obtained spectra from our method (NAM-IR) matches the IR spectra measured by an FTIR spectrometer. NAM-IR with the carbon string resonators provided more resolved spectra with a few ng of sample and faster sample preparation compared with traditional FTIR method requiring several $\mu g$ of drug. As a limitation, nanoparticles deposited with the jet nebulizer sampling method having a total mass of below ng could not be identified by NAM-IR. The pyrolytic carbon string resonators also showed the ability of in situ regeneration by local heating with the 
readout laser. We believe that the string resonators are promising for application in qualitative and quantitative monitoring in pharmaceutical manufacturing.

\section{Acknowledgement}

The research is funded by the Danish National Research Foundation (DNRF122) and Villum Fonden (Grant No. 9301). Center for Intelligent Drug delivery and Sensing Using Microcontainers and Nanomechanics (IDUN). This work was performed in part at DTU Danchip/Cen the National Center for Micro and Nanofabrication at the Technical University of Denmark.

\section{References}

[1] Zielinski, A. T., Prasad, A., Seshia, A. A., Kalberer, M., \& Jones, R. L. (2015). Effects of spatial sensitivity on mass sensing with bulk acoustic mode resonators. Sensors and Actuators A-Physical, 236, 369-379.

[2] Heinisch, M., Voglhuber-Brunnmaier, T., Reichel, E. K., Dufour, I., \& Jakoby, B. (2015). Electromagnetically driven torsional resonators for viscosity and mass density sensing applications. Sensors and Actuators A-Physical, 229, 182-191.

[3] Aoust, G., Levy, R., Bourgeteau, B., \& Le Traon, O. (2016). Acoustic damping on flexural mechanical resonators. Sensors and Actuators A-Physical, 238, 158-166.

[4] A. Boisen, S. Dohn, S.S. Keller, S. Schmid, M. Tenje, Cantilever-like micromechanical sensors, Rep. Prog. Phys. 4 (2011).

[5] E. Gil-Santos, D. Ramos, A. Jana, M. Calleja, A. Raman, J. Tamayo, Mass sensing based on deterministic and stochastic responses of elastically coupled nanocantilevers. Nano Lett. 9(12), 4122-4127 (2009)

[6] Larsen, T.; Schmid, S.; Gronberg, L.; Niskanen, A.; Hassel, J.; Dohn, S.; Boisen, A. , Ultrasensitive string-based temperature sensors. Appl. Phys. Lett. 2011, 98 (12) 121901- 121901/3

[7] Larsen, T.; Schmid, S.; Boisen, A., Micro string resonators as temperature sensors, AIP Conference Proceedings 2013, 931, 1552

[8] Schmid, S., Villanueva, L. G., \& Roukes, M. L. (2016). Fundamentals of nanomechanical resonators. Fundamentals of Nanomechanical Resonators, 1-175.

[9] Larsen, T.; Schmid, S.; Villanueva, L. G.; Boisen, A., Photothermal Analysis of Individual Nanoparticulate Samples Using Micromechanical Resonators ACS Nano 2013, 7 (7) 6188- 6193 
[10] Schmid, Silvan; Kurek, Maksymilian; Adolphsen, Jens Q; Boisen, Anja, Realtime single airborne nanoparticle detection with nanomechanical resonant filter-fiber, Scientific Reports, Vol. 3, 2013, p. 1288.

[11] Zhang, X.; Myers, E. B.; Sader, J. E.; Roukes, M. L., Nanomechanical Torsional Resonators for Frequency-Shift Infrared Thermal Sensing, Nano Lett. 2013, 13 (4) 1528- 1534

[12] Kim, S., Lee, D., Liu, X., Van Neste, C., Jeon, S., \& Thundat, T., Molecular recognition using receptor-free nanomechanical infrared spectroscopy based on a quantum cascade laser. Scientific Reports, 2013, 3(1), 1111.

[13] Dongkyu Lee, Seonghwan Kim, Thomas Thundat, Detection of biological analytes using nanomechanical infrared spectroscopy with a nanoporous microcantilever, Proc. SPIE 8879, Nano-Bio Sensing, Imaging, and Spectroscopy, 88790Q (20 May 2013)

[14] Anderson.D. Smith, Sam Vaziri, Frank Niklaus, Andreas.C. Fischer, Mikael Sterner, Anna Delin, Mikael Östling, Max.C. Lemme, Pressure sensors based on suspended graphene membranes, Solid-State Electronics, pp 89-94, Vol 88, 2013.

[15] M.A. Eltaher, M.A. Agwa, Analysis of size-dependent mechanical properties of CNTs mass sensor using energy equivalent model, Sensors and Actuators A: Physical, Volume 246, 2016, Pages 9-17.

[16] Malladi, K., Wang, C., Madou, M.: Fabrication of suspended carbon microstructures by e-beam writer and pyrolysis. Carbon N. Y. 44, 2602-2607 (2006).

[17] Beidaghi, M., Chen, W., Wang, C.: Electrochemically activated carbon microelectrode arrays for electrochemical micro-capacitors. J. Power Sources. 196, 24032409 (2011).

[18] Xu, H., Malladi, K., Wang, C., Kulinsky, L., Song, M., Madou, M.: Carbon postmicroarrays for glucose sensors. Biosens. Bioelectron. 23, 1637-1644 (2008).

[19] Teixidor, G.T., Gorkin, R. a, Tripathi, P.P., Bisht, G.S., Kulkarni, M., Maiti, T.K., Battacharyya, T.K., Subramaniam, J.R., Sharma, A., Park, B.Y., Madou, M.: Carbon microelectromechanical systems as a substratum for cell growth. Biomed. Mater. 3, 034116 (2008).

[20] Wang, C. L., \& Madou, M. (2005). From MEMS to NEMS with carbon. Biosensors and Bioelectronics, 20(10), 2181-2187.

[21] Martinez-Duarte, R. (2014). SU-8 Photolithography as a Toolbox for Carbon MEMS. Micromachines, 5(3), 766-782. 
[22] Kurek, Maksymilian; Larsen, Frederik K.; Larsen, Peter Emil; Schmid, Silvan; Boisen, Anja; Keller, Stephan Sylvest, Nanomechanical Pyrolytic Carbon Resonators: Novel Fabrication Method and Characterization of Mechanical Properties, Sensors, Vol. 16, No. 7, 2016, p. 1097.

[23] Quang, Long Nguyen; Larsen, Peter Emil; Boisen, Anja; Keller, Stephan Sylvest, Tailoring stress in pyrolytic carbon for fabrication of nanomechanical string resonators, Carbon, pp 358-368, vol 133, July 2018.

[24] Yamada, Shoko; Schmid, Silvan; Larsen, Tom; Hansen, Ole; Boisen, Anja, Photothermal Infrared Spectroscopy of Airborne Samples with Mechanical String Resonators, Analytical Chemistry, Vol. 85, No. 21, 2013, p. 10531-10535.

[25] R. A. Granberg and A. C. Rasmuson, Solubility of Paracetamol in Pure Solvents, Journal of Chemical Engineering Data, 1999, 44, 1391-1395.

[26] Bose, Sanjukta; Keller, Stephan Sylvest; Boisen, Anja; Almdal, Kristoffer; Alstrøm, Tommy Sonne, Process optimization of ultrasonic spray coating of polymer films, Langmuir, Vol. 29, No. 23, 2013, p. 6911-6919.

[27] Larsen, P. E., Boisen, A., \& Schmid, S. (2016). Hollow MEMS: An Integrated Sensor for Combined Density, Viscosity, Buoyant Mass and IR Absorption Spectrometry. DTU Nanotech.

[28] National Institute of Standards and Technology, NIST, U.S. Secretary of Commerce on behalf of the U.S.A. https://webbook.nist.gov/cgi/cbook.cgi?ID=C103902\&Type=IR-SPEC\&Index=1

[29] Prashanthi, K., Phani, A., \& Thundat, T. (2015). Photothermal Electrical Resonance Spectroscopy of Physisorbed Molecules on a Nanowire Resonator. Nano Letters, 15(8), 5658-5663. 